\title{
Milan 2020: COVID-19, neuro-oncology and much more
}

\author{
Gaetano Finocchiaro ${ }^{1}$
}

Received: 3 April 2020 / Accepted: 9 April 2020 / Published online: 13 April 2020

(c) Springer Science+Business Media, LLC, part of Springer Nature 2020

To the Editor,

In December 2019 one of our neurosurgeon colleagues at the Institution started suffering from flu-like symptoms. His respiratory capacity deteriorated and he was taken to the Emergency Unit of a specialized centre in Milan where he was connected to a ventilator. He eventually succumbed to the disease that initially was unnamed and that we now know as the consequence of COVID-19 infection.

Since then, this viral infection has spread dramatically in Northern Italy and specifically in Lombardy, affecting all aspects of our lives, personally and professionally [1].

As a neuro-oncologist, I am still working every day in my hospital but most of our contacts, with patients or colleagues, are mediated over the web. I spend a great part of my time analysing MRI scans that I receive digitally and trying to deal with difficult decisions, defining diagnoses and deciding on treatments with little or no possibility to interact directly with patients.

At the moment in Milan you are not allowed to walk around, you are advised to stay at home: all the stores are closed, except for alimentary markets, newsstands and few others and distance e-commerce is growing (Fig. 1). This decreases the chance for our patients, that are often immunesuppressed by their disease, steroids, radiotherapy or chemotherapy, to be exposed to COVID-19 but definitely their quality of life is significantly affected by the fear of facing this pandemic with an immune system that is significantly weakened.

However, we and the patients struggle to maintain all the possibilities open in terms of treatment and diagnosis. We doctors, nurses and the technicians in the hospital, still face this invisible danger but keep going as we should do, because this is our duty.

Gaetano Finocchiaro

gaetano.finocchiaro@istituto-besta.it

1 Unit of Molecular Neuro-Oncology, Fondazione IRCCS Istituto Neurologico Carlo Besta, 20133 Milan, Italy
On another level, we try to continue our research work in the field. Part of this can be done from home, writing and reading papers and analysing data. Experimental work, however, requires our presence in the labs and, in turn, that companies maintain their activities making reagents available and that transport is functioning.

But there is also another part of our lives that is touched by the pandemic: that part has to do with our role in society as doctors and scientists. A few days ago, while going to work, I met the caretaker of our building and had a chat with him: he mentioned to me that he and other inhabitants of the building felt safer because I was there and could help in case of emergency or just try to explain what was going on and, above all, make predictions as to the end of this situation or at least regarding its improvement. This role, our role is, of course, also important at home, with family members, with friends and sometimes with just bystanders. We have to champion the scientific approach: discourage the idea that COVID-19 is the result of a conspiracy, or even of bad science; trust in laboratory work (and, yes, animal experiments too!) aimed at identifying a vaccine (by the way, everyone who was anti-vax has strangely disappeared just recently); provide understandable explanations on numbers and graphs that each day are submerging us, from whatever source, be it the television, newspapers the web or the social media.

We also have to help politicians make informed choices, learn from the evidence and start looking ahead, not just run after the events. We did good things in Lombardy but we also made mistakes, and we and others will hopefully learn from these mistakes. One for all: avoid transforming hospitals into the problem rather than the remedy. Hospitalize patients that really need it and, above all, check all the operators with swabs, even if asymptomatic. They might be so because of their immune system, which for reasons now unknown is particularly fit to fight COVID-19, but the contagion to other unfit bystanders could become dangerous, even lethal. And now that the number of infected people may be platooning (here, of course), we need to set up reliable assays to determine IgM and IgG levels in the peripheral blood of people exposed to the virus. Once identified, these 


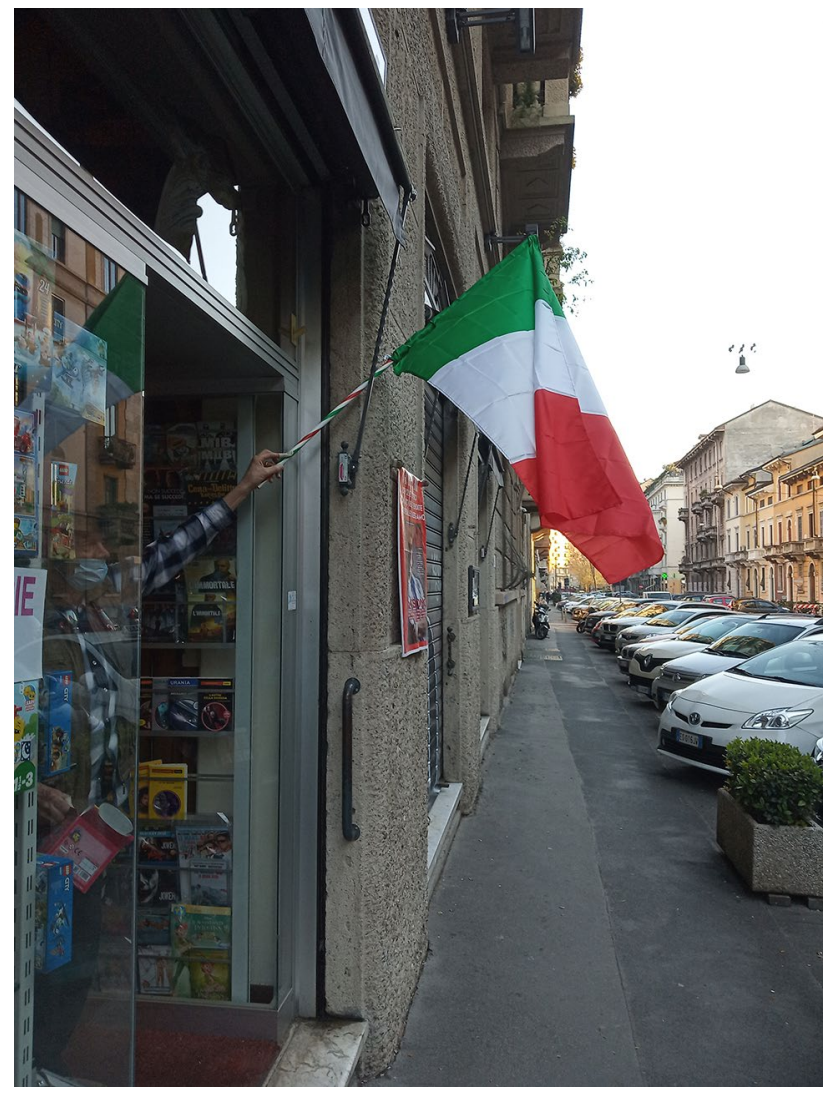

Fig. 1 April 2, 2020. A deserted street in Milan and the owner of a newsstand waving the Italian flag people will be the first to be able to come back to work and semi-normal life: which is absolutely necessary if we want to avoid, after a medical disaster, a social and economic disaster in our society.

A lot of commitments, many different layers of responsibility coherently with our roles as neuro-oncologists, doctors, scientists, and citizens challenged by such very difficult times.

\section{Compliance with ethical standards}

Conflict of interest The author declares that he/she has no conflict of interest.

Ethical approval This article does not contain any study with human participants performed by the author.

\section{Reference}

1. The Lancet Oncology (2020) COVID-19: global consequences for oncology. Lancet Oncol 21:467

Publisher's Note Springer Nature remains neutral with regard to jurisdictional claims in published maps and institutional affiliations. 